\title{
Nursing Practice in Recovery Room: A Case of Kibuye Hope Hospital and Mutoyi Hospital
}

\author{
Darius Niyungeko, Emmanuel Gasaba, Ariane Ndayisaba, Jean Bosco Ndikumana, \\ Justin Ndayisaba, Amina Uwamahoro, Angèle Nzobakenga, Esdras Nishimwe, \\ Margueritte Ndikumana, Rose Barbara*
}

Faculty of Health Sciences, Hope Africa University, Bujumbura, Burundi

Email: *barbara.rose@arbor.edu

How to cite this paper: Niyungeko, D., Gasaba, E., Ndayisaba, A., Ndikumana, J.B., Ndayisaba, J., Uwamahoro, A., Nzobakenga, A., Nishimwe, E., Ndikumana, M. and Barbara, R. (2021) Nursing Practice in Recovery Room: A Case of Kibuye Hope Hospital and Mutoyi Hospital. Open Journal of Nursing, 11, 139-151.

https://doi.org/10.4236/ojn.2021.113013

Received: February 21, 2021

Accepted: March 20, 2021

Published: March 23, 2021

Copyright $\odot 2021$ by author(s) and Scientific Research Publishing Inc. This work is licensed under the Creative Commons Attribution International License (CC BY 4.0).

http://creativecommons.org/licenses/by/4.0/

\section{(c) (i) Open Access}

\begin{abstract}
Background: Nursing care in the recovery room is oriented to take into account the patient's condition after surgery intervention with its main purpose of providing direct and continuous patient observation in emergence from general or regional anesthesia. In the absence of professional assistance, patients can develop complications that can lead them into shock or death. This study aims to understand the place of nurses in guiding nursing care in recovery room in two hospitals of Gitega Province by assessing the nurse's knowledge and attitudes for the promotion of quality nursing care for post-operative patients. Methods: A cross sectional study design was used to assess the practice of nurses in managing nursing care in the recovery room in these two hospitals. A purposive sampling method was used to select the 82 nurses working in the recovery room for these two hospitals and Alain Bouchard's formula was used to calculate the sample size. Data were collected using a self-report method involving questionnaire completion with five components addressing participants identifications, factors related to the work organization, factors related to the work environment, factors related to healthcare system, and nursing interventions in recovery room. Results: Findings revealed a significant lack of knowledge among the participants and their attitudes were slightly poor as for most of the variables of factors related to healthcare system as their score was less than $50 \%$ and their knowledge for nursing interventions was also poor as most of variable scores were less than $25 \%$. Conclusions: The study findings were slightly poor as their scores were less than $50 \%$ in most of the variables; therefore, it was recommended that in-service training and workshops should be organized by these healthcare facilities for the purpose of empowering the nurse's knowledge and practice. Moreover, these institutions should provide and encourage nurses to use nursing guidelines and protocols.
\end{abstract}




\section{Keywords}

Nursing Practice, Recovery Room, Nursing Guidelines, Postoperative Care, Postoperative Patient

\section{Introduction}

Nursing care in the recovery room is oriented to take into account the patient's condition after surgery intervention with its main purpose of providing direct and continuous patient observation in emergence from general or regional anesthesia. These latter allow healthcare professionals especially nurses to detect and restore disrupted patients' needs. Patient is the subject of monitoring and treating appropriately to his condition through oxygen therapy, ventilation, circulation, state of consciousness, and vital signs. According to WHO, nurses who are assigned in the recovery room must have a well-defined job description to meet the standards with specific objectives. In addition, they must provide nursing care independently in collaboration with patients, while integrating nursing theory in their practice for inpatients according to their disrupted needs [1]. However, in the absence of professional assistance, patients can develop complications that can lead them into shock or death. To overcome this challenge, it is necessary to take care of a post operated patient in holistic concepts, by taking into account its environment [2].

However, in Burundi, around 10\% of patients who have undergone surgery and who are in the recovery room are exposed to anesthetic accidents [3]. Some authors recommend to use guidelines relating to the Alderte score, Stewart score and Kremlin score in Bicêtre in which the elements of monitoring were developed to allow nurses to transfer patient inpatient department by following standardized quotation of the evaluation degree.

In developed countries, such as Europe, the United States, Australia, and New Zealand, nurses who work in the recovery room have required skills and were able to define clearly the nursing process according to national guidelines. This allows nurses to interpret and plan nursing care and decision making [4].

In developing countries such as South Africa, Cameroon and Nigeria, on the other hand, nurses working in recovery rooms are challenged by lack of necessary material resources, guidelines and skills to ensure quality care. Most patients in the recovery room experience complications as a result of their incompetence [5].

In Burundi, the management of recovery rooms is not established according to the guidelines required by the hospital manual and procedures, because each hospital provides care in accordance of its capacity [6]. After surgery in the operating room, patients are monitored in the recovering room for a period of 2 to 8 hours depending on the patient's condition [3]. Even though this surveillance exists and is assisted by healthcare providers (doctors and nurses), no study has 
been carried out to assess professional knowledge and attitudes, especially among nurses. In addition, nursing care is carried out by nurses without taking into account specific planning in the recovery room [6]. However, the worst can happen when caring for operated patients, especially since there are no national guidelines developed in Burundi for nursing in recovery room.

This study aims to understand the practice of nurses in guiding nursing care in recovery room in two hospitals of Gitega Province, namely Kibuye Hope hospital and Mutoyi Hospital by assessing the nurse's knowledge and attitudes for the promotion of quality nursing care for post-operative patients.

\section{Conceptual Framework}

This study used Orem's theory of care to direct professionals to focus primarily on the health of the patient as "human being". The professional has the capacities, skills and power to engage and perform self-care actions. Orem determines three nursing intervention systems which are designed to meet the patient's self-care needs according to the degree of disruption of their needs such as: the total help system, the partial help system and the support system and training. By applying her principles, such as administration of care, consideration of curative factors oriented on human needs, efficiency of care based on the promotion of health for the patient and his family, and his development, environment of the patient and the staff, the nursing response oriented towards the acceptance of the patient, the practices centered on nursing care taking into account the patient's condition [7].

By applying self-care theory in recovery room, it helps healthcare professionals to guide or orient their practice in holistic perspective of nursing care. Self-care is linked to the deviation of health status; thus, nurses have to do all the best to restore the patient's health. Identification of self-care deficits followed by surgical intervention for patients and its consequences can help the professional to restore his health system by acting on the priorities of self-care. This theory also allows professionals in recovery room to make a self-evaluation based on the quality improvement of patient. However, if nursing profession is oriented without considering theoretical concepts and definitions, the outcomes will be tiny. This is why Orem's theory will help nurses in structuring quality in their nursing practices in general and in recovery room in particular [8].

Literature review was generally reviewed on definition of keys concepts, history and importance of recovery room, current practice guidelines in the recovery room, preparation of the recovery room, equipment and facilities, nursing profile in recovery room, nurses' role in the recovery room, nursing process, nursing skills in recovery room, nurses' responsibilities in recovery room, discharge criteria in recovery room, the different elements of scores to be evaluated in recovery room (Aldrete Score, Stewart Score, Kremlin Score) and Criteria for maintaining motorized surveillance in the recovery room (Figure 1). 


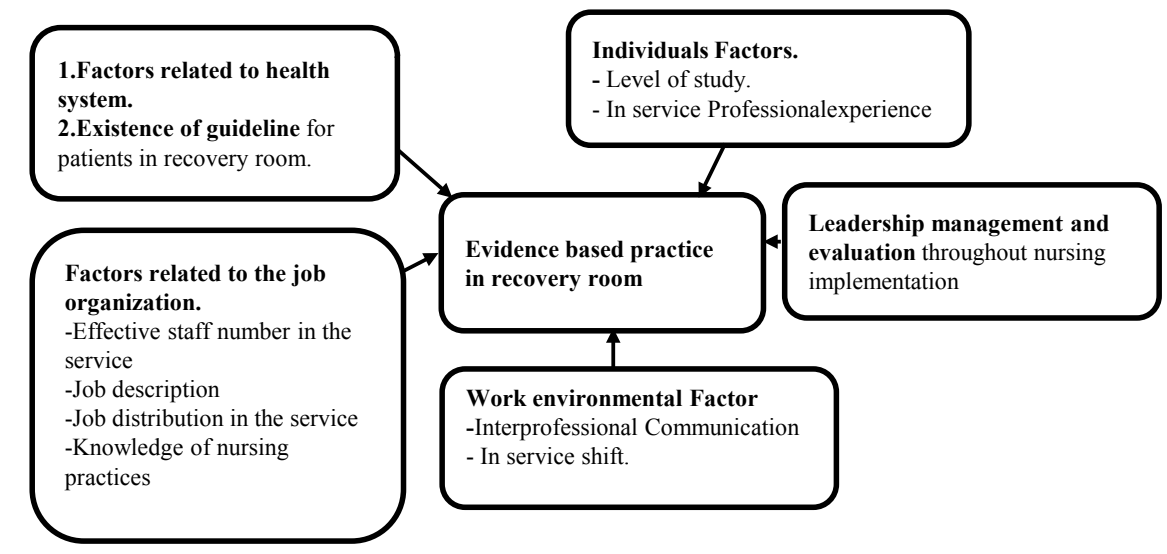

Figure 1. Conceptual framework for EBP in recovery.

\section{Methods}

Participants in this study were nurses working in these two districts hospitals among the five ones of Gitega province, in Burundi: Mutaho District Hospital and Kibuye District Hospital from September to November, 2019. These latter were chosen among those five because of their capacity as they serve a catchment population of 5479 patients with 205 beds and 5623 patients with 161 beds respectively for Mutoyi Hospital and Kibuye Hospital. A cross sectional study design was used to assess the practice of nurses in managing nursing care in the recovery room in these two hospitals of Gitega Province by assessing the nurse's knowledge and attitudes for the promotion of quality nursing care for post-operative patients. A purposive sampling method was used to select the 82 nurses who work in recovery room for these two hospitals. Nurses who did not worked in recovery rooms, those on leave and trainees were excluded from the study. Alain Bouchard's formula was used to calculate this sample of 82 nurses. The formula was:

$$
N C=\frac{n}{1+\frac{n}{N}}
$$

Kibuye Hope Hospital has the population of 68 nurses. By using Alain Bouchard's formula, the sample was 40 participants. $N C=\frac{96}{1+\frac{96}{68}}=39.8 \approx 40$

Mutoyi Hospital has the population of 75 nurses. By using Alain Bouchard's formula, the sample was 42 participants. $N C=\frac{96}{1+\frac{96}{75}}=42$

This is to make a total sample of 82 participants in this study. A self-report method involving questionnaire completion with five components addressing participants identifications, factors related to the work organization, factors related to the work environment, factors related to healthcare system, and nursing interventions in the recovery room. Furthermore, the questionnaire's validity and reliability were tested before being distributed to the participants and the 
findings from this latter had revealed also a poor knowledge and attitude towards nursing practice in recovery room. This preliminary study was conducted at Van Norman Clinic; a Free Methodist related institution located in Bujumbura city. The questionnaire was distributed only to those who were working in the recovery room. The investigator had to explain before the purpose of the study and after giving their verbal consent, participants were given a questionnaire to fill in. Questionnaires were filled in under investigators' supervision to avoid any collaboration during the completion to exclude bias. After being filled, questionnaires were collected for useful purpose. A Statistical Package for Social Scientists version 21.0 software (SPSS) was used to analyze the data which were presented as a frequency tables, bar graphs. The score of the results were classified refer to the items' scored by participants in each section of variables. It was good for those who scored more than $60 \%$, it was medium for those whose score were between $40 \%$ and $59 \%$ and for those whose score were less than $40 \%$ was classified as poor. Ethical principles had been respected where an oral informed consent was given to participants and these latter were given a choice whether to participate in the survey or not and the authorization to carry out the study was required from the University research committee and the hospitals superintendents from these institutions.

\section{Results}

The profile of nurses in recovery services in these two hospitals revealed that on the 82 nurses interviewed, $90 \%$ of participants were nurses with professional experiences which is oriented to patients needs in recovery room, and $80 \%$ were qualified nurses. Their ages were ranged between 23 years and 47 years $(\bar{x}=$ 32.3). Majority of them were female (67.1\%) while $32.9 \%$ were man (Table 1 ).

\subsection{Factors Related to the Organization of Work}

Concerning the factors related to the organization of work, the findings revealed that the majority $(85.37 \%)$ of participants had never had any in-service training. However, a significant number among participants argue that the 8 hours of working time are respected (93.9\%), human resources are fairly relocated into the service (63\%) and job description guidelines were available (59.80\%) (Figure 2).

Table 1. The participants' profile.

\begin{tabular}{|c|c|c|}
\hline \multicolumn{2}{|l|}{ Characteristics } & \multirow{2}{*}{$\begin{array}{c}\text { Frequency } \\
23-47 \text { years }\end{array}$} \\
\hline \multirow{2}{*}{ Age: } & Range & \\
\hline & Mean & 32.3 Years \\
\hline \multirow{2}{*}{ Sex } & Male & $27(32.9 \%)$ \\
\hline & Female & $55(67.1 \%)$ \\
\hline Nurses qualification/ & Yes & $80 \%$ \\
\hline Have a required diploma & No & $20 \%$ \\
\hline \multirow{2}{*}{ Nurses experiences to orient the patients' needs } & Yes & $90 \%$ \\
\hline & No & $10 \%$ \\
\hline
\end{tabular}




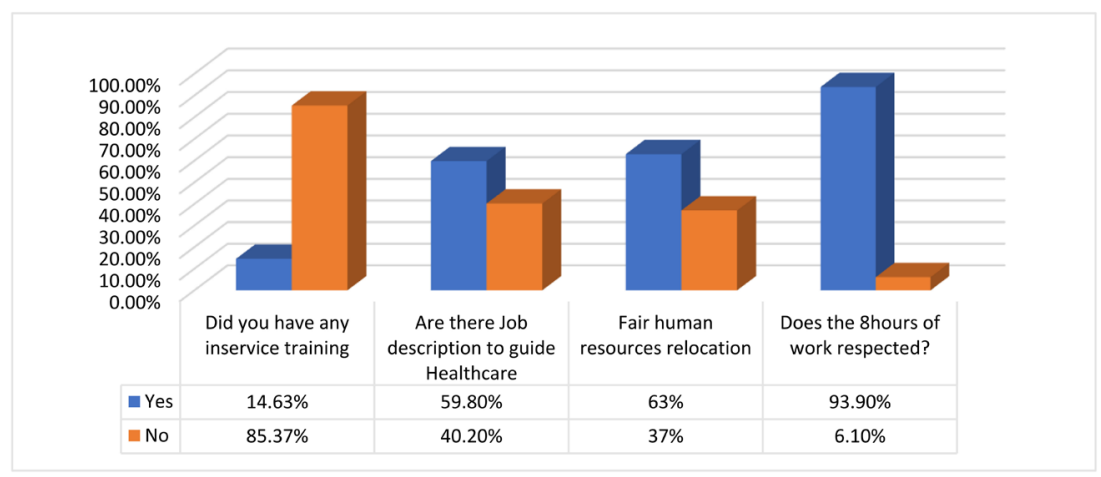

Figure 2. Factors related to the organization of work.

\subsection{Factors Related to the Work Environment}

Concerning factors related to work environment that facilitate nursing care, this study revealed that majority $(98.8 \%)$ of participants assumed that there is a good interprofessional collaboration between healthcare practitioners and adequate work condition in recovery room (78\%) (Table 2 ).

In this study, findings revealed that, in these two hospitals, all participants (100\%) assumed that there was no existence of any guidelines in recovery room to orient their nursing interventions. They additionally argued that they have never consulted anything as a nursing guideline used in this service. This study revealed also a poor knowledge among participants on different tools used to assess patient prior hospital discharge (less than $10 \%$ for all variables). Their knowledge was not too sufficient because only $47.56 \%$ were able to know the nursing protocols used for Post-Operative patient, $48.78 \%$ were the only ones who know the required time to transfer patients from recovery room to inpatient room and $51.22 \%$ assumed to be able to detect any complication that may arise in recovery room. Even though, all participants assumed that they know their roles in recovery room. Concerning their attitudes, majority of the participants $(87.80 \%)$, assumed that there is a good interprofessional collaboration, as nursing report could be shared between healthcare professionals, whilst $58.54 \%$ argued to respect the time for vital signs taking during nursing surveillance and monitoring (Table 3).

\subsection{Nursing Interventions}

Nursing interventions taken before the patient leaves the recovery room to their inpatient room revealed a quite deficiency knowledge among participants as majority of them did not respect patients' discharge criteria (93.9\%), 87.8\% do not respect the patients monitoring interventions, $85.3 \%$ did not wash their hand before and after nursing interventions, $82.65 \%$ had never had any workshop on nursing intervention in recovery room and $73.17 \%$ argue that patients are kept more than 6 hours lying down. However, participants revealed a significant knowledge on patients' immobilization and air way management to avoid post-operative complication like bed sores and up air way obstruction (Figure 3). 


\section{Discussions}

Nursing in recovery room requires integration and strategies based on different theory-based approaches to nursing and professionals must be able to analyze deeply the patients' data which is mentioned in the patient files as well as the assessment of priorities according to their needs while deciding on the nursing protocol. Nursing standards documents must be followed during the nursing implementation for better implementing nursing quality care.

Table 2. Factors related to the work environment.

\begin{tabular}{ccc}
\hline Characteristics & & Frequency \\
\hline Interpersonal collaboration & Yes & $98.8 \%$ \\
Working conditions & No & $1.2 \%$ \\
& Yes & $78 \%$ \\
\hline
\end{tabular}

Table 3. Factors related to healthcare system.

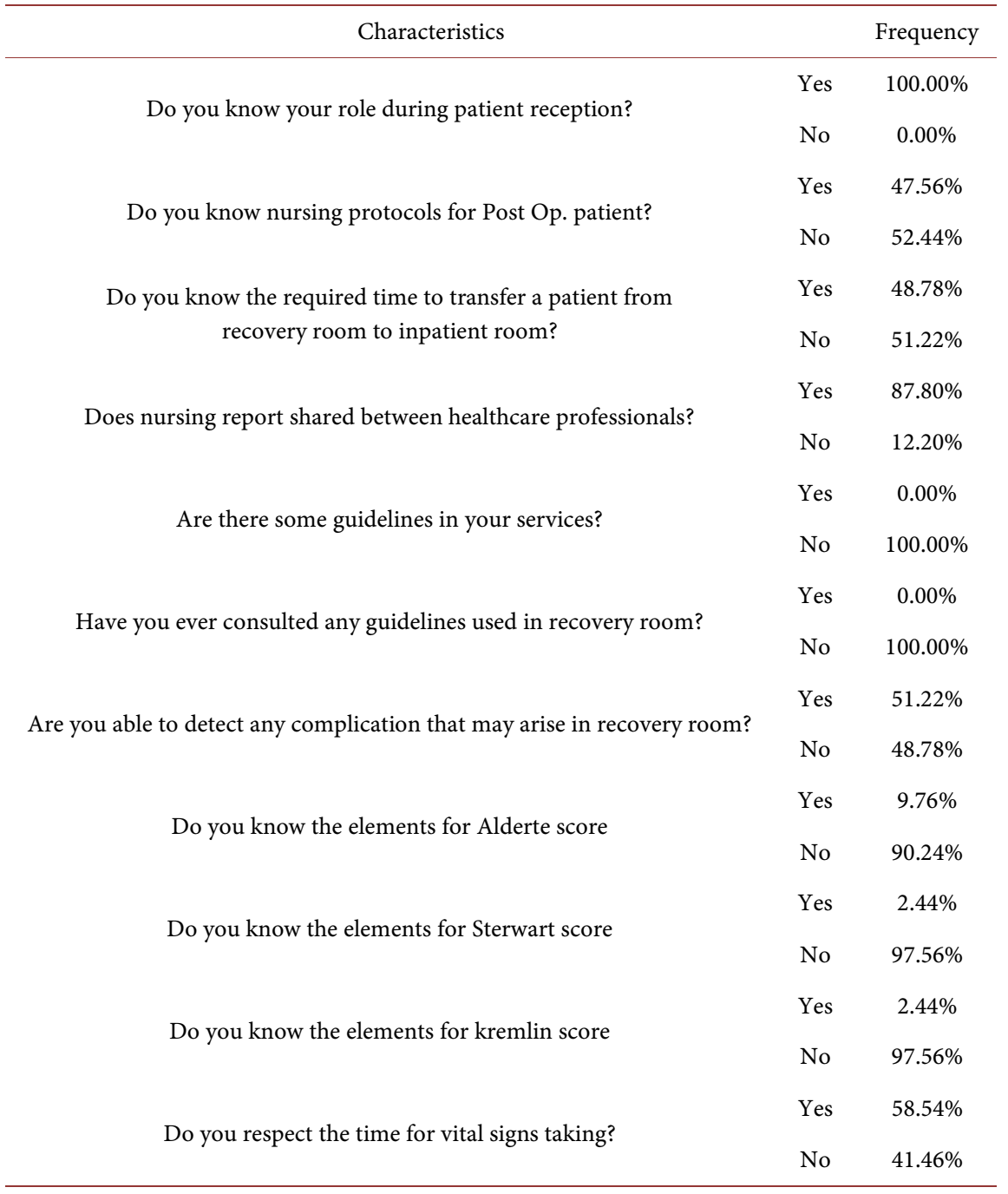




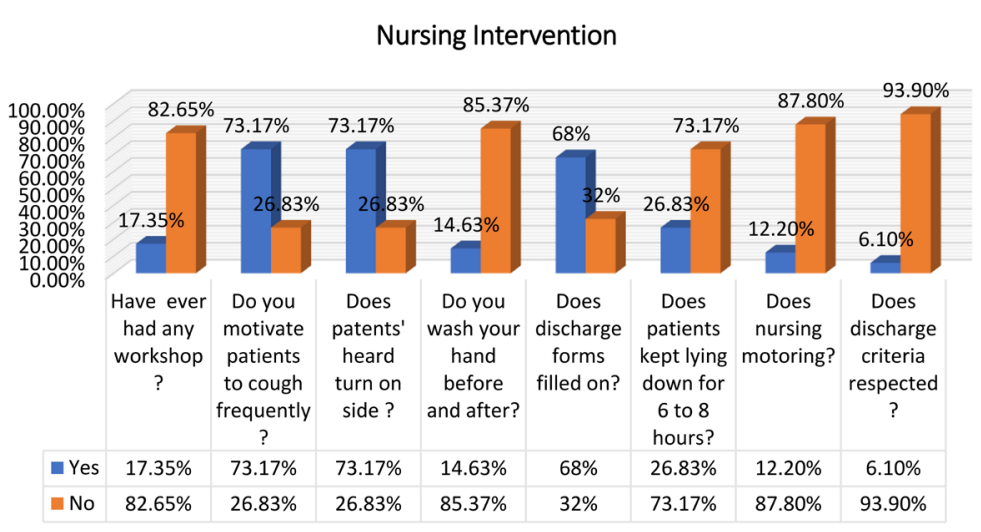

Figure 3. Nursing interventions taken before the patient is discharged from the recovery room to their inpatient room.

The findings in these two hospitals revealed that the ages of the participants were ranged between 23 years and 47 years $(\bar{x}=32.3)$ and majority of them were female (67.1\%). $90 \%$ of participants were nurses with professional experiences which are oriented to patients needs in recovery room and $80 \%$ were qualified nurses (Table 1). In their study Dimitry D \& Feruza I. (2009) suggested that there should be sufficient trained staff to allow continuous one-to-one care of every patient passing through the recovery room. In developed country it required one nurse for one patient in recovery room [9]. Therefore, the nurse practitioner responsibility for the care of a patient in the recovery room is to prevent complications, detect early complications, relieve patient's discomfort, support patients through their state of dependence to independence, and closely monitor the patient's condition which are most skills that these professionals nurse gain trough their nursing training which would be reinforced by regular in-service training or during their workshop.

Concerning the factors related to the organization of work, the findings revealed that majority (85.37\%) of participants had never had any in service training which can be very dangerous for the patient. Recovery room is a special nursing unit that accommodates a group of patients who have just undergone major or minor surgery and its purpose is to provide direct and continuous patient observation during emergence from general or regional anesthesia. This concurs with the findings of Van Huyssteen \& Botha (2004) where they argued that in most developing countries such as South Africa, Cameroon and Nigeria nurses working in recovery rooms are challenged by lack of necessary material resources, guidelines and skills to ensure quality care [5]. This could help these latter to provide effective quality care especially if it was guided by research. It could be better also for nurse managers to organize in-service training especially for those who did not yet get it, for better updating their knowledge. In their study, they argued also that in-service training is the most crucial tool for quality improvement of in-patient nursing care, because empowering education could facilitate occupational tasks and achieve greater mastery of nursing professional 
skills. Therefore, in-service training is the expected requirement to enhance the effectiveness of nursing care [10].

However, a significant number among participants argue that the 8 hours of working time are respected (93.9\%), human resources are fairly relocated into the service (63\%) and job description guidelines were available (59.80\%) (Figure 2). Therefore, evidence suggests that nurse staffing affects the quality of care in hospitals especially in recovery room where care is addressed to critical patients. Moreover, findings from 168 Pennsylvania hospitals showed that every time there enter a new patient in recovery room, they are an added average workload for staff registered nurses (RNs) and can increased the risk of death following common surgical procedures by $7 \%$. The risk of death was more than $30 \%$ higher in hospitals where nurses' mean workloads were 8 patients or more each shift than in hospitals where nurses cared for 4 or fewer patients [11]. Therefore, this could not be the case in these two hospitals and in Burundian hospitals in general to reach this level of one patient to one professional nurse due to this country's status as a low-income sub-Saharan economy. However, by making schedules of 8-hour shifts, fair relocation of human resources, well-defined job description and guidelines could prevent significantly these post-operative risks in recovery room.

Concerning factors related to work environment that facilitate nursing care, this study revealed that majority (98.8\%) of participants assumed that there is a good interprofessional collaboration between healthcare practitioners and adequate work condition in recovery room (78\%) (Table 2). This concours with the findings of Débora C. et al. 2009 where they argued that the multi-professional team that engaged with post-operative patients has the objectives of offering support to patients during the anesthetic recovery period up until the patient reaches cardiorespiratory stability and conscience; preventing or treating eventual complications; providing measures to relieve post operatory pain where nurses are required to render a safe, rational and individualized assistance; and providing the patient with support throughout his return to a normal post-anesthetic physiological status [12]. Moreover, Walt JH in her study argued recovery rooms must therefore be seen to be as important as the operating theatre or intensive care unit and should reflect quality of the staff, design of the area, and facilities. It should be purpose-designed and located adjacent to or within the operating theatre complex. Unrestricted access for medical staff from inside and outside the operating suite is essential to maintain supervision and care [13].

In this study, findings revealed that, in these two hospitals, all participants $(100 \%)$ assumed that there was no existence of any guidelines in recovery rooms to orient their nursing interventions. They additionally argued that they have never consulted anything as a nursing guideline used in this service. In addition to lack of in-service training (Figure 2), these HCFs do not provide guidelines for nursing care in recovery rooms. This could be a health threat to patients with probability of high risks of mortality, healthcare cost and elongated hospital stays. Therefore, it could be better for these institutions to monitor their service 
functions, implement and promote the use of guidelines and evidence-based practice for better patients' outcomes. Discharging a post-operative patient from recovery room to surgical ward encompasses consideration of range criteria; therefore, guidelines can provide a consistent high standard of nursing care for all patients, reducing risks and put patient's safety and comfort at the center of nursing practice [14].

This study revealed also a poor knowledge among participants on different tools used to assess patient post-anesthesia discharge (less than $10 \%$ for all variables) (Table 3). This could confirm that in these two hospital nurses don't use any of the Alderte, Sterwart or Kremlin score documents. The effect of transferring patients from recovery room to in-patient unit without a thorough assessment of patients using these tools could result in high risk of developing other complications following this transfer. In her work, Laura P. D. (2015) argued that the use of a standardized tool provides consistency of care by reducing errors, promoting efficient use of resources, meets Joint Commission requirements, and meets ASPAN (American Society of Post-Anesthesia Nurses) recommended standards [15]. Dimitry D., et al. 2009 argued also that the modified Aldrete score is a simple sum of numerical values assigned to activity, respiration, circulation, consciousness, and oxygen saturation by including also the assessment of pain, nausea/vomiting, and surgical bleeding in addition to vital signs and activity [9]. Therefore, in-service training for these latter is required to enhance their patient's care and prevent all kinds of risks that could occur due to lack of knowledge in their nursing assessment and evaluation.

Their knowledge was not sufficient because only $47.56 \%$ were able to know the nursing protocols used for post-operative patients, $48.78 \%$ were the only ones who know the required time to transfer patients from recovery room to inpatient room and $51.22 \%$ assumed to be able to detect any complication that may arise in recovery room. This could be confirmed by the same problem of lack of in-service training, in-service guidelines and the spirit of research. Literature revealed that most of patients remain an average of 2 - 3 hours in recovery unit [12]. Moreover, to overcome these problems an academic content concerning the peri-operatory nursing area should be maintained in nursing graduation curriculum, guaranteeing proper work conditions and encouraging specialization courses to be taken on new proposals and trends, thus stimulating research that can improve patients' outcomes.

Even though, all participants assumed that they know their roles in recovery room. This could be explained by the fact that as they attend the patients, they believe that they know their role which is not correlated with their knowledge and practice in the above variables. Concerning their attitudes, a majority of the participants $(87.80 \%)$, assumed that there is a good interprofessional collaboration, as nursing report could be shared between healthcare professionals, whilst $58.54 \%$ argued to respect the time for vital signs take during nursing surveillance and monitoring (Table 3). Findings revealed that the most essential elements 
that nurses believed to improve their patients' experiences of quality nursing care could be clinically competent nurses, collaborative working relationships, autonomous nursing practice, adequate staffing, control over nursing practice, managerial support and patient-centered culture [16].

Nursing interventions taken before the patient leaves the recovery room to their inpatient room revealed a quite deficiency knowledge among participants as a majority of them did not respect patients' discharge criteria (93.9\%), 87.8\% did not respect the patients monitoring interventions, $85.3 \%$ did not wash their hand before and after nursing interventions, $82.65 \%$ had never had any workshop on nursing intervention in recovery room and $73.17 \%$ argue that patients are kept more than 6 hours lying down. This could be explained by the fact that these HCFs do not have any guidelines to orient their nursing interventions (Figure 2). To overcome all these challenges, the nurse manager should organize the services' activities by setting protocols and providing guidelines for nursing interventions in recovery room, organize workshop and in-services training so that their practices become evidence based on better outcomes of the patients; all these by promoting a spirit of research and documentations among healthcare providers. This concurs with the finding of Helen R. (2003) where she argued that to safely discharge patients from recovery room, they must provide practical guidance and advice to all staff responsible for nursing care of patients for the purpose of ensuring that consistent high standard nursing care is provided [14]. However, participants revealed a significant knowledge on patients' immobilization and air way management to avoid post-operative complication like bed sores and up air way obstruction (Figure 3 ).

\section{Recommendations}

The following are the recommendations that originated from the study:

- The administration of these two hospitals has to come up with strategies of updating the nurses' knowledge in nursing care of post-operative patients which is evidence based by integrating nursing theory and research into their practice. In-service training and workshops were recommended.

- The nurse manager should be encouraged in collaboration with the hospital's administration to put protocols and manual guidelines for nursing intervention in recovery room.

- University should revise the curriculum and include the skills related to the implementation of nursing practice which is evidence based.

- The government should allow the university to implement a curriculum plan for nursing specialty for advanced nursing practice.

\section{Conclusion}

This study revealed that recovery-room nurses lacked important knowledge and held poor attitudes related to their practice. Many scores related to factors of the healthcare system were scored less than $50 \%$ in the negative direction (Table 3 ). 
Moreover, knowledge for nursing interventions taken before the patient is discharged from the recovery room to their inpatient room was also poor as most of variable's scores were less than 25\%; however, participants revealed a significant knowledge on patients' immobilization and air way management to avoid post-operative complication like bed sores and up air way obstruction $(73.17 \%)$. Providing better curriculum, in-service training and recovery-room guidelines can greatly improve care to patients in the recovery room.

\section{Limitations}

The limitations of study were that our study was conducted in two rural hospitals among the five of Gitega province which is one in the eighteen ones of the country. It was also done in one unit (recovery room) and a limited number of nurses were interviewed.

\section{Conflicts of Interest}

The authors declare no conflicts of interest regarding the publication of this paper.

\section{References}

[1] University of Pittsburgh Medical Center (2012) What to Expect: Post Anesthesia Care Unit.

https://www.upmc.com/-/media/upmc/locations/hospitals/shadyside/services/surgi cal-services/post-anesthesia-care-unit/documents/pacu-brochure-upmc-shadyside. pdf

[2] Arias-Botero, J.H. and Padrón-Mercado, C.M. (2017) Portrayal of the Performance of the Post-Anesthesia Care Unit, Based on the Perception of the Professional Nursing Staff. Colombian Journal of Anesthesiology, 45, 16-23. https://doi.org/10.1016/j.rcae.2017.09.008

[3] MSPLS (2018) Statestique Annuelle. Ministère de la Santé Publique Burundi, Gitega, 48-76.

[4] Mccallum, J. (2015) Knowledge of Post-Operative Airway Emergencies in Recovery Room Nurses in Selected Hospitals.

http://wiredspace.wits.ac.za/jspui/bitstream/10539/21223/1/FinalSubmissionJuly201 5.pdf

[5] van Huyssteen, T. and Botha, A. (2004) Recovery room Nurses' Knowledge Regarding Postoperative Airway Emergencies in Adults in Private Hospitals in Northern Gauteng, South Africa. Southern African Journal of Anaesthesia and Analgesia, 10, 9-13. https://doi.org/10.1080/22201173.2004.10872370

[6] PNDS II (2014) Rapport d'évaluation du plan national de développement deuxième génération.

https://www.prb.org/wp-content/uploads/2020/06/Burundi-Plan-National-de-D\%C 3\%A9veloppement-Sanitaire-2011-2015.pdf

[7] Charles, F. (2011) Concepts fondateurs de la démarche soignante: Modèles et courants de pensée.

http://ifsi.charlesfoix.blog.free.fr/public/Concepts_fondateurs_de_la_demarche_soi gnante.pdf 
[8] Fawcett, J. (2001) The Nurse Theorists: 21st-Century Updates-Dorothea E. Orem. Nursing Science Quarterly, 14, 34-38.

https://journals.sagepub.com/doi/abs/10.1177/08943180122108021 https://doi.org/10.1177\%2F08943180122108021

[9] Dimitry, D. and Feruza, I. (2009) Recovery Room: Safety Island in the Operating Theatre. Medical Journal of Zambia, 36, 132-135.

https://www.ajol.info/index.php/mjz/article/view/56079/44534 https://doi.org/10.4314/mjz.v36i3.56079

[10] Chaghari, M., Saffari, M., Ebadi, A. and Ameryoun, A. (2017) Empowering Education: A New Model for In-Service Training of Nursing Staff. Journal of Advances in Medical Education \& Professionalism, 5, 26-32.

https://www.ncbi.nlm.nih.gov/pmc/articles/PMC5238493/

[11] Aiken, L.H., Clarke, S.P., Cheung, R.B., Sloane, D.M. and Silber, J.H. (2003) Educational Levels of Hospital Nurses and Surgical Patient Mortality. JAMA, 290, 1617-1623. https://jamanetwork.com/journals/jama/fullarticle/197345 https://doi.org/10.1001/jama.290.12.1617

[12] Débora, C. and Aparecida, P. (2009) Nurse Interventions and the Complications in the Post-Anesthesia Recovery Room. Revista da Escola de Enfermagem da USP, 43, 953-961.

http://www.scielo.br/scielo.php?script=sci_arttext\&pid=S0080-62342009000400030 \&lng=en\&nrm=iso\&tlng=en

[13] Walt, J.H. (1988) Patient Safety in the Recovery Room. Anaesthesia and Intensive Care, 16, 77-80. https://doi.org/10.1177\%2F0310057X8801600126 https://journals.sagepub.com/doi/pdf/10.1177/0310057X8801600126

[14] Helen, R. (2003) Criteria for the Safe Discharge of Patients from the Recovery Room. NursingTimes, 99, 22.

https://www.nursingtimes.net/clinical-archive/public-health-clinical-archive/criteri a-for-the-safe-discharge-of-patients-from-the-recovery-room-23-09-2003/

[15] Dowling, L.P. (2015) Aldrete Discharge Scoring: Appropriate for Post Anesthesia Phase I Discharge? Master's Theses of Science, University of New Hampshire, Durham. https://scholars.unh.edu/thesis/14

[16] Renate, K., Brigitte, B., Anneke, L.F. and Diana, D. (2014) How Nurses and Their Work Environment Affect Patient Experiences of the Quality of Care: A Qualitative Study. BMC Health Services Research, 14, Article No. 249. https://bmchealthservres.biomedcentral.com/articles/10.1186/1472-6963-14-249 https://doi.org/10.1186/1472-6963-14-249 